\title{
BRAID FAMILY REPRESENTATIVES
}

\author{
SLAVIK JABLAN and RADMILA SAZDANOVIĆ ${ }^{\dagger}$ \\ The Mathematical Institute, Knez Mihailova 35, \\ P.O.Box 367, 11001 Belgrade, \\ Serbia \& Montenegro \\ jablans@mi.sanu.ac.yu \\ seasmile@galeb.etf.bg.ac.yu ${ }^{\dagger}$
}

\section{ABSTRACT}

After defining reduced minimum braid word and criteria for a braid family representative, different braid family representatives are derived, and a correspondence between them and families of knots and links given in Conway notation is established.

\section{Introduction}

In the present article Conway notation $[1,2,3,4]$ will be used without any additional explanation. A braid-modified Conway notation is introduced in Section 1, for a better understanding of the correspondence between braid family representatives $(B F R \mathrm{~s})$ and families of knots and links $(K L \mathrm{~s})$ given in Conway notation.

Minimum braids are defined, described, generated and presented in tables for knots up to ten crossings and oriented links up to nine crossings by T. Gittings [5]. T. Gittings used them for studying graph trees, amphicheirality, unknotting numbers and periodic tables of $K L \mathrm{~s}$.

Since knots are 1-component links, the term $K L$ will be used for both knots and links.

In Section 2 we define a reduced braid word, describe general form for all reduced braid words with $s=2$ strands, generate all braid family representatives of twostrand braids, and establish a correspondence between them and families of $K L \mathrm{~s}$ given in Conway notation. In Section 3 we consider the same problem for $s \geq 3$. In Section 4 some applications of minimum braids [5] and braid family representatives are discussed. All computations are made using the knot-theory program LinKnot written by the authors [6], the extension of the program Knot2000 by M. Ochiai and N. Imafuji [7].

\section{Reduced Braid Words and Minimum Families of Braids with $s=2$}

We use the standard definition of a braid and description of minimum braids given by T. Gittings [5]. Instead of $a \ldots a$, where a capital or lower case letter $a$ appears $p$ times, we write $a^{p} ; p$ is the degree of $a(p \in N)$. It is also possible to work with negative powers, satisfying the relationships: $A^{-p}=a^{p}, a^{-p}=A^{p}$. A 
number of strands is denoted by $s$, and a length of a braid word by $l$.

The operation $a^{2} \rightarrow a$ applied on any capital or lower case letter $a$ is called idempotency. To every braid word we can apply the operation of idempotency until a reduced braid word is obtained.

Definition 1. A reduced braid word is a braid word with degree of every capital or lower case letter equal to 1.

By an opposite procedure, braid word extension, from every reduced braid word we obtain all braid words that can be derived from it by assigning a degree (that can be greater then 1) to every letter. In this case, a reduced braid word plays a role of a generating braid word.

A braid word with one or more parameters denoting degrees greater then one represents a family of braid words. If values of all parameters are equal 2, it will be called a source braid.

For the minimality of reduced braids we are using the following criteria:

1. minimum number of braid crossings;

2. minimum number of braid strands;

3. minimum binary code for alternating braid crossings.

According to the first and second criterion minimal reduced braids are the shortest reduced braids with a smallest as possible number of different letters among all equivalent reduced braids representing certain $K L$. A binary code for any braid crossing can be generated by assigning a zero for an alternating, and a one for a non-alternating crossing. Hence, a priority will be given to alternating braids, and then to braids that differ from them as low as possible. Analogous minimality criteria can be applied to source braids.

Definition 2. Among the set of all braid families representing the same $K L$ family, the braid family representative $(M F B)$ is the one that has the following properties:

1. minimum number of braid crossings;

2. minimum reduced braid;

3. minimum source braid.

These criteria are listed in descending order of importance for determining $B F R$ s.

Our definition of $B F R$ s results in some fundamental differences with regard to minimum braids, defined by T. Gittings [5]. Some members of $B F R$ s will be minimum braids, but not necessarily.

For example, the minimum braid of the link .2 $1: 2\left(9_{11}^{3}\right.$ in Rolfsen [4]) is 9:03-05a $A A b A C b A C b$ [5, Table 2]. According to the second $B F R$ criterion it will be derived 
from the generating minimum braid $A b A b A C b C$ corresponding to the link $.21\left(8_{13}^{2}\right)$, and not from the non-minimum generating braid $A b A C b A C b$ corresponding to the same link. Hence, to the three-component link .21:2(9 $\left.9_{11}^{3}\right)$ obtained as the first member of $B F R A b A^{p} b A C b C$ for $p=2$ will correspond the braid $A b A A b A C b C$, that is not a minimum braid according to the minimum braid criteria [5].

The third criterion: minimum source braid enables us to obtain $K L \mathrm{~s}$ of a certain family from a single $B F R$, and not from several different $B F R \mathrm{~s}$. For example, applying this criterion, $K L \mathrm{~s} .3 .2 .20, .2 .3 .20$ and .2 .2 .30 belonging to the same $K L$ family .r.p. $q 0$ will be obtained from the single $B F R A^{p} b A^{q} b A b^{r}$. Otherwise, using the minimum braid criteria [5], the knot .3.2.2 0 will be obtained from the family $A^{p} b A b^{q} A b^{r}$, three-component link .2 .3 .20 will be obtained from $A^{p} b A^{q} b A b^{r}$, and the knot .2 .2 .30 will be obtained from $A^{p} b A^{q} b^{r} A b$ for $p=3, q=2, r=2$. Source braids corresponding to the families $A^{p} b A b^{q} A b^{r}, A^{p} b A^{q} b A b^{r}$ and $A^{p} b A^{q} b^{r} A b$ are $A^{2} b A b^{2} A b^{2}, A^{2} b A^{2} b A b^{2}$ and $A^{2} b A^{2} b^{2} A b$, respectively, and the second source braid is minimal. Hence, the representative of the $K L$ family .r.p.q 0 is $B F R A^{p} b A^{q} b A b^{r}$.

According to this, to every $B F R$ can be associated a single corresponding family of $K L \mathrm{~s}$ given in Conway notation and vice versa.

An overlapping of $K L$ families obtained from $B F R$ s can occur only at their beginnings. For example, distinct $B F R \mathrm{~s} A b A^{p} b A C b C$ and $A^{p} b C b A b C b$, giving $K L$ families .21:p and .p $1: 2$, respectively, for $p=2$ will have as a joint member aforementioned three-component link .21:2(9 $\left.9_{11}^{3}\right)$. According to the second $B F R$ criterion, it will be derived from the minimum generating braid $A b A b A C b C$, and not from $A b A C b A C b$. Hence, $B F R A b A^{p} b A C b C$ giving $K L \mathrm{~s}$ of the form $.21: p$ begins for $p=2$, and $A^{p} b C b A b C b$ giving $K L \mathrm{~s}$ of the form for $. p 1: 2$ begins for $p=3$. In this way, all ambiguous cases can be solved.

Every $K L$ is algebraic (if its basic polyhedron is $1^{*}$ ) or polyhedral, so according to this criterion, all $K L \mathrm{~s}$ are divided into two main categories: algebraic and polyhedral. Since to every member of a $B F R$ corresponds a single $K L$, we can introduce the following definition:

Definition 3. An alternating $B F R$ is polyhedral iff its corresponding $K L$ s are polyhedral. Otherwise, it is algebraic. A non-alternating $B F R$ is polyhedral iff its corresponding alternating $B F R$ is polyhedral. Otherwise, it will be called algebraic.

The division of non-alternating $B F R$ s into algebraic and polyhedral does not coincide with the division of the corresponding $K L \mathrm{~s}[1,2,3]$, because minimum number of braid crossings is used as the first criterion for the BFRs. Accepting minimum reduced braid universe [5] as the first criterion, all $K L$ s derived from the basic polyhedron .1 will be algebraic, because they can be represented by non-alternating minimal (but not minimum [5]) algebraic braids. E.g., the alternating knot 2.20 $\left(8_{16}\right)$ with the polyhedral braid $A^{2} b A^{2} b A b$ can be represented as the algebraic knot $(-3,2)(3,-2)$ with the corresponding algebraic braid $A^{3} b^{2} a^{2} B^{3}$. In this case, to the knot 816 corresponds algebraic braid $A^{3} b^{2} a^{2} B^{3}$ that reduces to $A b a B$, and not $A^{2} b A^{2} b A b$ that reduces to $A b A b A b$. 
Another solution of this discrepancy is changing the definition of an algebraic $K L$ into the following:

Definition 4. $K L$ is algebraic if it has an algebraic minimum crossing number representation.

In this case, all $K L$ s derived from the basic polyhedron .1 (with Conway symbols beginning with a dot) will be polyhedral $K L \mathrm{~s}$, because their minimum crossing number representations are polyhedral.

We will consider only $B F R$ s corresponding to prime $K L \mathrm{~s}$.

It is easy to conclude that every 1-strand $B F R$ is of the form $A^{p}$, with the corresponding $K L$ family $p$ in Conway notation.

Theorem 1. Every reduced $B F R$ with $s=2$ is of the form $(A b)^{n}, n \geq 2$.

This $B F R$ corresponds to the knot 22 and to the family of basic polyhedra $.1=6^{*}, 8^{*}, 10^{*}, 12^{*}$ (or 12A according to A. Caudron [3]), etc. For $n \geq 3$ all of them are $n$-antiprisms. Let us notice that the first member of this family, the knot 22 , is not an exception: it is an antiprism with two digonal bases.

Theorem 2. All algebraic alternating $K L \mathrm{~s}$ with $s=2$ are the members of the following families:

$p 12$ with the $B F R A^{p} b A b(p \geq 1)$;

$p 11 q$ with the $B F R A^{p} b A b^{q}(p \geq q \geq 2)$;

$p, q, 2$ with the $B F R A^{p} b A^{q} b(p \geq q \geq 2)$;

$p, q, r 1$ with the $B F R A^{p} b A^{q} b^{r}(r \geq 2, p \geq q \geq 2)$;

$(p, r)(q, s)$ with the $B F R A^{p} b^{q} A^{r} b^{s}$

$(p, q, r, s \geq 2, p \geq r, p \geq s, s \geq q$ and if $p=s$, then $r \geq q)$.

Minimum braids include one additional braid $\left(A^{p} b^{q} A b^{r}\right)$ in the case of algebraic alternating $K L$ s with $s=2$.

Alternating polyhedral $K L$ s with $s=2$ are given in the following table, each with its BFR. KLs in this table are given in "standard" Conway notation (that is "standardized" for knots with $n \leq 10$ and links with $n \leq 9$ crossings according to Rolfsen's book [4]). This table can be extended to an infinite list of antiprismatic basic polyhedra $(2 n)^{*}$ described by the $B F R \mathrm{~s}(A b)^{n}, n \geq 3$ and $B F R \mathrm{~s}$ with $s=2$ obtained as their extensions.

Table 1

Basic polyhedron $.1=6^{*}$

$\begin{array}{llllll}A^{p} b A b A b & . p & (1) & A^{p} b A b A^{q} b^{r} & r: p 0: q 0 & (7) \\ A^{p} b A b A b^{q} & . p . q & (2) & A^{p} b A b^{q} A^{r} b^{s} & \text { p.s.r.q } & (8) \\ A^{p} b A^{q} b A b & . p . q 0 & (3) & A^{p} b A^{q} b A^{r} b^{s} & q 0 . p . r 0 . s 0 & (9)\end{array}$




\begin{tabular}{|c|c|c|c|}
\hline$A^{p} b A b^{q} A b$ & $. p: q 0$ & $A^{p} b A^{q} b^{r} A b^{s}$ & .p.s.r $0 . q 0$ \\
\hline$A^{p} b A^{q} b A b^{r}$ & $. r . p . q 0$ & $A^{p} b A^{q} b^{r} A^{s} b^{t}$ & p.t.s.r.q \\
\hline$A^{p} b A^{q} b A^{r} b$ & $p: q: r$ & $A^{p} b^{q} A^{r} b^{s} A^{t} b^{u}$ & p.q.r.s.t.u \\
\hline
\end{tabular}

If we apply minimum braid criteria [5], we need to add ten braids for the basic polyhedron $.1=6^{*}$ : (1') $A^{p} b A b^{q} A b^{r}$, (2') $A^{p} b A^{q} b^{r} A b$, (3') $A^{p} b^{q} A b A b^{r}$, (4') $A^{p} b A^{q} b^{r} A^{s} b,\left(5^{\prime}\right) A^{p} b^{q} A b A^{r} b^{s},\left(6^{\prime}\right) A^{p} b^{q} A b^{r} A b^{s},\left(7^{\prime}\right) A^{p} b^{q} A^{r} b A b^{s},\left(8^{\prime}\right) A^{p} b^{q} A b^{r} A^{s} b^{t}$, (9') $A^{p} b^{q} A^{r} b A^{s} b^{t}$, (10') $A^{p} b^{q} A^{r} b^{s} A b^{t}$. Applying $B F R$ criteria, according to the minimum source braid criterion all $K L$ s obtained from the braids (1') and (2') will be obtained from $B F R(5), K L$ s obtained from (3') will be obtained from (7), KLs obtained from (4') and (6') will be obtained from (9), $K L$ s obtained from (5') and $\left(7^{\prime}\right)$ will be obtained from (8), and $K L$ s obtained from ( $\left.8^{\prime}\right),\left(9^{\prime}\right)$ and (10') will be obtained from (11). Using minimum braid criteria [5], we need to make analogous additions to all classes of $B F R$ s considered in this paper.

For the basic polyhedron $8^{*}$ we have:

\section{Basic polyhedron $8^{*}$}

\begin{tabular}{|c|c|c|c|}
\hline$A^{p} b A b A b A b$ & $8^{*} p$ & $A^{p} b A^{q} b A b^{r} A b^{s}$ & $8^{*} p: q: . r: s$ \\
\hline$A^{p} b A b A b A b^{q}$ & $8^{*} p \cdot q$ & $A^{p} b A b^{q} A^{r} b A b^{s}$ & $8^{*} p . s \quad: \quad . r . q$ \\
\hline$A^{p} b A^{q} b A b A b$ & $8^{*} p: q$ & $A^{p} b A^{q} b A^{r} b A^{s} b$ & $8^{*} p: s: r: q$ \\
\hline$A^{p} b A b A b^{q} A b$ & $8^{*} p \quad: \quad . q$ & $A^{p} b A b A^{q} b^{r} A^{s} b^{t}$ & $8^{*}$ p.t.s.r.q \\
\hline$A^{p} b A b A^{q} b A b$ & $8^{*} p \quad:: \quad q$ & $A^{p} b A^{q} b A b^{r} A^{s} b^{t}$ & $8^{*}$ p.t.s.r $: \quad . q$ \\
\hline$A^{p} b A^{q} b A b A b^{r}$ & $8^{*} p . r \quad:: \quad . q$ & $A^{p} b A^{q} b^{r} A^{s} b A b^{t}$ & $8^{*} p:$ q.r.s : .t \\
\hline$A^{p} b A b A^{q} b A b^{r}$ & $8^{*} p . r: q$ & $A^{p} b A^{q} b A^{r} b A^{s} b^{t}$ & $8^{*}$ p.t.s $: r: q$ \\
\hline$A^{p} b A^{q} b A^{r} b A b$ & $8^{*} p: q: r$ & $A^{p} b A^{q} b A^{r} b^{s} A b^{t}$ & $8^{*}$ p.t : s.r : $q$ \\
\hline$A^{p} b A^{q} b A b^{r} A b$ & $8^{*} p: . r: . q$ & $A^{p} b A b^{q} A^{r} b^{s} A^{t} b^{u}$ & $8^{*}$ p.u.t.s.r.q \\
\hline$A^{p} b A b A b A^{q} b^{r}$ & $8^{*} p \cdot r \cdot q$ & $A^{p} b A^{q} b A^{r} b^{s} A^{t} b^{u}$ & $8^{*}$ p.u.t.s.r : $q$ \\
\hline$A^{p} b A b A b^{q} A^{r} b^{s}$ & $8^{*}$ p.s.r.q & $A^{p} b A^{q} b^{r} A^{s} b A^{t} b^{u}$ & $8^{*}$ p.u.t : s.r.q \\
\hline$A^{p} b A^{q} b A b A^{r} b^{s}$ & $8^{*}$ p.s.r :: $q$ & $A^{p} b A^{q} b^{r} A^{s} b^{t} A b^{u}$ & $8^{*} p: q . r . s . t: u$ \\
\hline$A^{p} b A b^{q} A^{r} b^{s} A b$ & $8^{*} p:$ : s.r.q & $A^{p} b A^{q} b^{r} A^{s} b^{t} A^{u} b^{v}$ & $8^{*}$ p.v.u.t.s.r.q \\
\hline$A^{p} b A^{q} b^{r} A b A b^{s}$ & $8^{*} p . s:: \quad r . q$ & $A^{p} b^{q} A^{r} b^{s} A^{t} b^{u} A^{v} b^{w}$ & $8^{*}$ p.q.r.s.t.u.v.w \\
\hline$A^{p} b A^{q} b A^{r} b A b^{s}$ & $8^{*} p . s: . r: q$ & & \\
\hline
\end{tabular}

Trying to better understand the correspondence between BFRs and Conway symbols of $K L \mathrm{~s}$, we can introduce modified Conway notation. Most of $K L \mathrm{~s}$ can be given in Conway notation by several different symbols (and this is the main disadvantage of Conway notation). In a similar way as with the classical notation, where every $K L$ is given by its place in knot tables, we need to use some "standard" code, according to the notation introduced in the original Conway's paper [2] and in the papers or books following it $[1,3,4]$. For example, the same polyhedral knot .$p$ can be given by ..p, : $p,: . p, \ldots$, or even as $6^{*} p, 6^{*} . p, 6^{*}: . p, \ldots$ 
Working with BFRs we introduce a braid-modified Conway notation that will be more suitable for denoting $K L$ s obtained from $B F R \mathrm{~s}$. We are trying to have a same degree $p$ at the first position of a braid, and as the first element of Conway symbol corresponding to it. Whenever possible, the order of degrees will be preserved in the corresponding Conway symbol. By using this notation, we can recognize a very simple pattern for $B F R$ s derived from the generating minimum braids of the form $(A b)^{n}$ : by denoting in a Conway symbol corresponding to a given braid every sequence of single letters of a length $k$ by $k+1$ dots, we obtain the Conway symbol of a given braid. In order to recognize this pattern for $K L \mathrm{~s}$ derived from basic polyhedra, first we need to use only one basic polyhedron $6^{*}$ with $n=6$ crossings, and not two of them $\left(.1\right.$ and $\left.6^{*}\right)$. In this case, the Table 1 will look as follows:

\section{Basic polyhedron $6^{*}$}

\begin{tabular}{|c|c|c|c|}
\hline$A^{p} b A b A b$ & $6^{*} p$ & $A^{p} b A b A^{q} b^{r}$ & $6^{*} p$ :: $q . r$ \\
\hline$A^{p} b A b A b^{q}$ & $6^{*} p \quad:: \quad . q$ & $A^{p} b A b^{q} A^{r} b^{s}$ & $6^{*} p:$ : q.r.s \\
\hline$A^{p} b A^{q} b A b$ & $6^{*} p: q$ & $A^{p} b A^{q} b A^{r} b^{s}$ & $6^{*} p: q: r . s$ \\
\hline$A^{p} b A b^{q} A b$ & $6^{*} p: . q$ & $A^{p} b A^{q} b^{r} A b^{s}$ & $6^{*} p: q . r: s$ \\
\hline$A^{p} b A^{q} b A b^{r}$ & $6^{*} p: q: . r$ & $A^{p} b A^{q} b^{r} A^{s} b^{t}$ & $6^{*} p:$ q.r.s.t \\
\hline$A^{p} b A^{q} b A^{r} b$ & $6^{*} p: q: r$ & $A^{p} b^{q} A^{r} b^{s} A^{t} b^{u}$ & $6^{*}$ p.q.r.s.t.u \\
\hline
\end{tabular}

and for the basic polyhedron $8^{*}$ we have:

\section{Basic polyhedron $8^{*}$}

\begin{tabular}{|c|c|c|c|}
\hline$A^{p} b A b A b A b$ & $8^{*} p$ & $A^{p} b A^{q} b A b^{r} A b^{s}$ & $8^{*} p: q: . r: s$ \\
\hline$A^{p} b A b A b A b^{q}$ & $::: \quad . q$ & $A^{p} b A b^{q} A^{r} b A b^{s}$ & $8^{*} p: . q . r: . s$ \\
\hline$A^{p} b A^{q} b A b A b$ & $8^{*} p: q$ & $A^{p} b A^{q} b A^{r} b A^{s} b$ & $8^{*} p: q: r: s$ \\
\hline$A^{p} b A b A b^{q} A b$ & $8^{*} p \quad:: \quad . q$ & $A^{p} b A b A^{q} b^{r} A^{s} b^{t}$ & $8^{*} p \quad:: \quad$ q.r.s.t \\
\hline$A^{p} b A b A^{q} b A b$ & $8^{*} p \quad:: \quad q$ & $A^{p} b A^{q} b A b^{r} A^{s} b^{t}$ & $8^{*} p: q:$.r.s.t \\
\hline$A^{p} b A^{q} b A b A b^{r}$ & $8^{*} p: q:: . r$ & $A^{p} b A^{q} b^{r} A^{s} b A b^{t}$ & $8^{*} p:$ q.r.s : .t \\
\hline$A^{p} b A b A^{q} b A b^{r}$ & $8^{*} p:: q: . r$ & $A^{p} b A^{q} b A^{r} b A^{s} b^{t}$ & $8^{*} p: q: r: s . t$ \\
\hline$A^{p} b A^{q} b A^{r} b A b$ & $8^{*} p: q: r$ & $A^{p} b A^{q} b A^{r} b^{s} A b^{t}$ & $8^{*} p: q: r . s: t$ \\
\hline$A^{p} b A^{q} b A b^{r} A b$ & $8^{*} p: q: . r$ & $A^{p} b A b^{q} A^{r} b^{s} A^{t} b^{u}$ & $8^{*} p:$.q.r.s.t.u \\
\hline$A^{p} b A b A b A^{q} b^{r}$ & $8^{*} p \quad::: \quad$ q.r & $A^{p} b A^{q} b A^{r} b^{s} A^{t} b^{u}$ & $8^{*} p: q:$ r.s.t.u \\
\hline$A^{p} b A b A b^{q} A^{r} b^{s}$ & $8^{*} p$ :: .q.r.s & $A^{p} b A^{q} b^{r} A^{s} b A^{t} b^{u}$ & $8^{*} p:$ q.r.s : t.u \\
\hline$A^{p} b A^{q} b A b A^{r} b^{s}$ & $8^{*} p: q:: r . s$ & $A^{p} b A^{q} b^{r} A^{s} b^{t} A b^{u}$ & $8^{*} p: q . r . s . t: u$ \\
\hline$A^{p} b A b^{q} A^{r} b^{s} A b$ & $8^{*} p:$ : q.r.s & $A^{p} b A^{q} b^{r} A^{s} b^{t} A^{u} b^{v}$ & $8^{*} p$ : q.r.s.t.u.v \\
\hline$A^{p} b A^{q} b^{r} A b A b^{s}$ & $8^{*} p: q \cdot r:: s$ & $A^{p} b^{q} A^{r} b^{s} A^{t} b^{u} A^{v} b^{w}$ & $8^{*}$ p.q.r.s.t.u.v.w \\
\hline$A^{p} b A^{q} b A^{r} b A b^{s}$ & $8^{*} p: q: r: . s$ & & \\
\hline
\end{tabular}

Unfortunately, it is not possible to express every family of $K L \mathrm{~s}$ in the braidmodified Conway notation. Another problem is that it strongly differs from the standard Conway notation. Therefore, the braid-modified Conway notation is used 
only when after some slight modification standard Conway symbols remained completely understandable to a reader familiar with them.

In the same way, it is possible to continue with the derivation of $B F R$ s from basic polyhedra with a higher number of crossings.

Hence, we conclude that:

Corollary All alternating $K L$ s with $s=2$ are described by Theorem 2 and by an infinite extension of Table 1.

From alternating $B F R \mathrm{~s}$ we obtain non-alternating $B F R \mathrm{~s}$ by crossing changes. This way, from $B F R$ s derived from the generating minimum braid $(A b)^{2}$ we obtain the following families of non-alternating $B F R$ s and corresponding new $K L$ families:

$\begin{array}{llll}A^{p} B a B & (p-1) 3 & A^{p} b a^{q} b^{r} & p,(q-1) 1,-(r+1) \\ A^{p} b a^{q} b & p,(q-1) 1,-2 & A^{p} B A^{q} B^{r} & p, q,-r 1 \\ A^{p} B A^{q} B & p, q,-2 & A^{p} B^{q} a^{r} B^{s} & (-p, r)(q, s) \\ A^{p} B a B^{q} & (p-1) 2 q & A^{p} B^{q} A^{r} B^{s} & (p, r)-(q, s) \\ A^{p} B^{q} a B^{r} & p-1, q, r+ & & \end{array}$

In the same way, we can derive non-alternating $B F R \mathrm{~s}$ with $s=2$ from the generating $B F R(A b)^{n}, n \geq 3$.

\section{Braid Family Representatives with $s \geq 3$}

In order to continue derivation of $B F R$ s and corresponding $K L \mathrm{~s}$ for $s \geq 3$ first we derive all different reduced minimum braid words. It is possible to establish general construction rules for generating minimum braid words.

Definition 5. For a given generating minimum braid word $W=w L$ that ends with a capital or lower case letter $L$, a replacement of $L$ by a word $w_{1}$ in $W$ will be called extending by replacement. An addition of the word $w_{1}$ to $W$ is extending by addition. The both operations are extending operations.

Definition 6. Let $W=w L_{s}$ and $w_{1}=L_{s+1} L_{s} L_{s+1}$ be generating minimum braids with $s$ and $s+1$ strings, where $L_{s}$ denotes $s$ th letter and $L_{s+1}$ denotes $(s+1)$ th letter. The word extending operations obtained this way will be called, respectively, $(s+1)$ extending by replacement, and $(s+1)$-extending by addition. The both operations are $(s+1)$-extending operations.

For example, the first operation applied on $A b A b$ gives $A b A C b C$, and the other $A b A b C b C$.

The $(s+1)$-extending by replacement is sufficient for construction of generating minimum braids for a given $s$, with $l=2 s$, corresponding to $K L \mathrm{~s}$ of the form $2 \ldots 2=2^{s}$, where 2 occurs $s$ times. For $2 \leq s \leq 6$ as the result we obtain: $A b A b$, $A b A C b C, A b A C b d C d, A b A C b d C E d E, A b A C b d C E d f E f \ldots$

The generating minimum braids for given $s$, with $l=3 s-2$, corresponding to $K L$ s of the form $21 \ldots 12=21^{3 s-6} 2$, where 1 occurs $3 s-6$ times, can be obtained 
using only $(s+1)$-extension by addition. For $3 \leq s \leq 6$ we obtain: $A b A b C b C$, $A b A b C b C d C d, A b A b C b C d C d E d E, A b A b C b C d C d E d E f E f \ldots$

Applying the same procedure, from $A^{3}$ we obtain the series $A^{3} B a B, A^{3} B a B C b C$, $A^{3} B a B C b C D c D, A^{3} B a B C b C D c D E d E \ldots$, corresponding to the knots $32,52,72$, $92 \ldots$

Analogously, starting with $w_{1}=A b A b C b d C d$ and using the $(s+1)$-extension by replacement, the generating minimum braids with $l=2 s+1$, corresponding to $K L$ s of the form $221 \ldots 12=2^{2} 1^{2 s-5} 2$ are obtained for given $s$.

However, in order to exhaust all possibilities, all combinations of $(s+1)$-extending operations are used for derivation of reduced minimum braids.

Theorem 3. Every generating algebraic minimum braid can be derived from $A b A b$ by a recursive application of $(s+1)$-extending operations.

The minimal generating braid words for $s \leq 5$ with their corresponding $K L \mathrm{~s}$ are given in the following table:

$\begin{array}{llll}s=1 & l=1 & A & 1 \\ s=2 & l=4 & A b A b & 22 \\ & & & \\ s=3 & l=6 & A b A C b C & 22 \\ s=3 & l=7 & A b A b C b C & 21112 \\ & & & \\ s=4 & l=8 & A b A C b d C d & 222 \\ s=4 & l=9 & A b A b C b d C d & 221112 \\ s=4 & l=10 & A b A b C b C d C d & 2111112 \\ & & & \\ s=5 & l=10 & A b A C b d C E d E & 2222 \\ s=5 & l=11 & A b A b C b d C d E d E & 2221112 \\ s=5 & l=11 & A b A C b C d C E d E & 2211122 \\ s=5 & l=12 & A b A b C b C d C E d E & 22111112 \\ s=5 & l=12 & A b A b C b d C d E d E & 211121112 \\ s=5 & l=13 & A b A b C b C d C d E c E & 2111111112\end{array}$

In the case of polyhedral generating minimum braid words it is also possible to make generalizations. We have already considered the infinite class of generating polyhedral minimum braid words $(A b)^{n}$ with $s=2$. The first infinite class with $s=3$ will be $(A b)^{n-1} A C b C$, with the corresponding $K L$ s of the form $(2 n)^{*} 210$.

Every $B F R$ can be derived from a generating minimum braid by assigning a degree (that can be greater then 1) to every letter.

For $s=3$ there are two generating alternating algebraic minimum braid words: $A b A C b C, l=6$, with the corresponding link 222 ;

$A b A b C b C, l=7$, with the corresponding knot 21112 , that generate prime $K L \mathrm{~s}$. 
From $A b A C b C$ we derived 17 alternating $B F R$ s and their corresponding families of $K L \mathrm{~s}$, given in the following table:

$\begin{array}{llll}A^{p} b A C b C & p 122 & A^{p} b A^{q} C b^{r} C & (p, q)(r, 2+) \\ A b A C b^{p} C & p, 2,2+ & A^{p} b^{q} A^{r} C b^{s} C & (p, r)(q, 2, s) \\ A^{p} b A C b^{q} C & p 1, q, 2+ & A^{p} b^{q} A C b^{r} C^{s} & p 1, q, s 1, r \\ A^{p} b A^{q} C b C & p, q, 22 & A^{p} b A^{q} C b^{r} C^{s} & (p, q)(r, s 1+) \\ A^{p} b A C b C^{q} & p 121 q & A^{p} b A^{q} C^{r} b C^{s} & (p, q) 2(r, s) \\ A b^{p} A C b^{q} C & p, 2, q, 2 & A^{p} b^{q} A^{r} C b^{s} C^{t} & (p, r)(q, t 1, s) \\ A^{p} b^{q} A C b^{r} C & p 1, q, r, 2 & A^{p} b A^{q} C^{r} b^{s} C^{t} & (p, q), s,(t, r)+ \\ A^{p} b A C b^{q} C^{r} & p 1, q, r 1+ & A^{p} b^{q} A^{r} C^{s} b^{t} C^{u} & (p, r), q,(u, s), t \\ A^{p} b A^{q} C b C^{r} & p, q, r 12 & & \end{array}$

The next generating alternating algebraic minimum braid $A b A b C b C$ of the length 7 , with $s=3$, gives the following results:

$\begin{array}{llll}A^{p} b A b C b C & p 11112 & A^{p} b^{q} A b C b^{r} C^{s} & (p 1, q) 1(s 1, r) \\ A b A b C b^{p} C & p, 211,2 & A^{p} b A^{q} b^{r} C b^{s} C & (p, q) 1 r(2, s) \\ A b A b^{p} C b C & 21 p 12 & A^{p} b A^{q} b^{r} C b C^{s} & p, q, s 11 r 1 \\ A^{p} b^{q} A b C b C & p 1, q, 211 & A^{p} b A^{q} b C^{r} b C^{s} & (p, q) 111(r, s) \\ A^{p} b A^{q} b C b C & p, q, 2111 & A^{p} b A^{q} b C b^{r} C^{s} & (p, q) 11(r, s 1) \\ A^{p} b A b^{q} C b C & p 11 q 12 & A^{p} b A b^{q} C b^{r} C^{s} & p 11 q, r, s 1 \\ A^{p} b A b C b C^{q} & p 11111 q & A^{p} b A b C^{q} b^{r} C^{s} & (p 111, r)(q, s) \\ A b A b^{p} C b^{q} C & 21 p, q, 2 & A^{p} b^{q} A^{r} b C b^{s} C & (p, r), q,(2, s) 1 \\ A b^{p} A b C b^{q} C & (p, 2) 1(q, 2) & A^{p} b^{q} A b^{r} C b^{s} C & (p 1, q) r(2, s) \\ A^{p} b^{q} A^{r} b C b C & (p, r)(q, 211) & A^{p} b^{q} A^{r} b C b^{s} C^{t} & (p, r), q,(t 1, s) 1 \\ A^{p} b^{q} A b^{r} C b C & p 1, q, 21 r & A^{p} b^{q} A b^{r} C b^{s} C^{t} & (p 1, q) r(t 1, s) \\ A^{p} b^{q} A b C b^{r} C & (p 1, q) 1(r, 2) & A^{p} b A^{q} b^{r} C^{s} b C^{t} & (p, q) 1 r 1(s, t) \\ A^{p} b A^{q} b C b^{r} C & (p, q) 11(2, r) & A^{p} b A^{q} b^{r} C b^{s} C^{t} & (p, q) 1 r(s, t 1) \\ A^{p} b A^{q} b^{r} C b C & p, q, 21 r 1 & A^{p} b A^{q} b C^{r} b^{s} C^{t} & (p, q) 11,(t, r), s \\ A^{p} b A^{q} b C b C^{r} & p, q, r 1111 & A^{p} b A b^{q} C^{r} b^{s} C^{t} & (p 11 q, s)(r, t) \\ A^{p} b A b^{q} C b^{r} C & p 11 q, r, 2 & A^{p} b^{q} A^{r} b^{s} C b^{t} C & (p, r), q,(t, 2) s \\ A^{p} b A b^{q} C b C^{r} & p 11 q 11 r & A^{p} b^{q} A^{r} b^{s} C b^{t} C^{u} & ((p, r), q) s(u 1, t) \\ A^{p} b A b C b^{q} C^{r} & p 111, q, r 1 & A^{p} b^{q} A^{r} b C^{s} b^{t} C^{u} & ((p, r), q) 1((u, s), t) \\ A b^{p} A b^{q} C b^{r} C & (p, 2) q(r, 2) & A^{p} b A^{q} b^{r} C^{s} b^{t} C^{u} & (p, q) 1 r((u, s), t) \\ A^{p} b^{q} A^{r} b^{s} C b C & (p, r)(q, 21 s) & A^{p} b^{q} A^{r} b^{s} C^{t} b^{u} C^{v} & ((p, r), q) s((u, t), v)\end{array}$

Except $A b A C b C$ and $A b A b C b C$, all generating minimum braids with $s=3$ are polyhedral.

For $s=3$ and $l \leq 12$, the polyhedral generating braids and their corresponding $K L$ s are given in the following table, with the notation for basic polyhedra with 12 crossings according to A. Caudron [3]:

$\begin{array}{llllll}l=8 & A b A b A C b C & .21 & l=11 & A b A b A b A b C b C & 8^{*} 211 \\ l=8 & A b C b A b C b & .2: 2 & l=11 & A b A b A b C b C b C & 11^{* * *} \\ & & & l=11 & A b A b A C b A b C b & 10^{* *} .20 \\ l=9 & A b A b C b A b C & 8^{*} 20 & l=11 & A b A b A C b A C b C & 11^{* *}\end{array}$


Braid Family Representatives

$\begin{array}{llllll}l=9 & A b A b A b C b C & .211 & l=11 & A b A b C b A C b C b & 11^{*} \\ l=9 & A b A C b A C b C & 9^{*} & & & \\ & & & l=12 & A b A b A b A b A C b C & 10^{*} 210 \\ l=10 & A b A b A b C b C b & .212 & l=12 & A b A b A b A C b C b C & 12 \mathrm{I} \\ l=10 & A b A b A b A C b C & 8^{*} 210 & l=12 & A b A b A b C b A C b C & 12 \mathrm{~F} \\ l=10 & A b A b A C b A b C & 9^{*} .2 & l=12 & A b A b A C b A b C b C & 12 \mathrm{H} \\ l=10 & A b A b C b A b C b & 9^{*} 2 & l=12 & A b A b C b A b A C b C & 12 \mathrm{G} \\ l=10 & A b A b A C b C b C & 10^{* * *} & l=12 & A b A b C b A b C b C b & 12 \mathrm{D} \\ l=10 & A b A b C b A C b C & 10^{* *} & l=12 & A b C b A b C b A b C b & 12 \mathrm{C}\end{array}$

From them, $B F R$ s without duplications are derived. E.g., for $l=8$, the generating minimum braid .21 gives $70 B F R \mathrm{~s}$, and $.2: 2$ gives $19 B F R \mathrm{~s}$. Overlapping of those families can occur only if all parameters are equal 2 , i.e., for source braids and source $K L \mathrm{~s}$ corresponding to them. According to the minimality criteria, all those source braids will belong to the first $B F R$. The generating minimum braid .21 gives the following $B F R$ s:

\begin{tabular}{|c|c|c|c|}
\hline$A^{p} b A b A C b C$ & $.21 . p 0$ & $A b A^{p} b A C^{q} b C^{r}$ & $. p:(q, r) 1$ \\
\hline$A b A^{p} b A C b C$ & $.21: p$ & $A b A^{p} b^{q} A C b^{r} C$ & $. p . q .(2, r)$ \\
\hline$A b A b A C b^{p} C$ &.$(p, 2)$ & $A b A^{p} b^{q} A C b C^{r}$ & $r 11 . q . p$ \\
\hline$A b A b A C b C^{p}$ &.$p 11$ & $A b A b^{p} A C b^{q} C^{r}$ & $p \cdot(r 1, q)$ \\
\hline$A b A b^{p} A C b C$ & $.21 . p$ & $A b A b^{p} A C^{q} b C^{r}$ & $. p .(r, q) 1$ \\
\hline$A^{p} b A^{q} b A C b C$ & $.21 . p 0 . q$ & $A b^{p} A^{q} b^{r} A C b C$ & 2 1.p.r $0 . q 0$ \\
\hline$A^{p} b A b A^{q} C b C$ & $210: p 0: q 0$ & $A b^{p} A b^{q} A C b^{r} C$ & $p: q:(2, r) 0$ \\
\hline$A^{p} b A b A C b^{q} C$ &.$(2, q) \cdot p 0$ & $A b^{p} A b^{q} A C b C^{r}$ & $q: p: r 110$ \\
\hline$A^{p} b A b A C b C^{q}$ & $q 11 . p 0$ & $A^{p} b A^{q} b A^{r} C b^{s} C$ & $(2, s) \cdot p 0 \cdot r \cdot q 0$ \\
\hline$A^{p} b A b^{q} A C b C$ & $. q .21 . p 0$ & $A^{p} b A^{q} b A^{r} C b C^{s}$ & $s 110: r 0 . q . p 0$ \\
\hline$A^{p} b^{q} A b A C b C$ & $21: p: q 0$ & $A^{p} b A^{q} b A C b^{r} C^{s}$ & $.(s 1, r) \cdot p 0 . q$ \\
\hline$A b A^{p} b A C b^{q} C$ & $(2, q): p$ & $A^{p} b A^{q} b A C^{r} b C^{s}$ & $(s, r) 1: p \cdot q 0$ \\
\hline$A b A^{p} b A C b C^{q}$ & $q 11: p$ & $A^{p} b A^{q} b^{r} A^{s} C b C$ & q.r.s.210.p \\
\hline$A b A^{p} b^{q} A C b C$ & $.21 . q . p$ & $A^{p} b A^{q} b^{r} A C b^{s} C$ & $(s, 2) \cdot r . q \cdot p 0$ \\
\hline$A b A b A C b^{p} C^{q}$ & $(q 1, p)$ & $A^{p} b A^{q} b^{r} A C b C^{s}$ & $. s 11 . r . q . p 0$ \\
\hline$A b A b A C^{p} b C^{q}$ & $(q, p) 1$ & $A^{p} b A b A^{q} C b^{r} C^{s}$ & $(r, s 1) 0: q 0: p 0$ \\
\hline$A b A b^{p} A C b^{q} C$ &.$(q, 2) \cdot p$ & $A^{p} b A b A^{q} C^{r} b C^{s}$ & $(s, r) 10: q 0: p 0$ \\
\hline$A b A b^{p} A C b C^{q}$ & $. q 11 . p$ & $A^{p} b A b^{q} A^{r} C b^{s} C$ & $q .(2, s) \cdot r 0 . p$ \\
\hline$A b^{p} A b^{q} A C b C$ & $p: q: 210$ & $A^{p} b A b^{q} A^{r} C b C^{s}$ & q.s $11 . r 0 . p$ \\
\hline$A^{p} b A^{q} b A^{r} C b C$ & $.21 . p 0 . q: r$ & $A^{p} b A b^{q} A C b^{r} C^{s}$ & $q \cdot(s 1, r) \cdot p 0$ \\
\hline$A^{p} b A^{q} b A C b^{r} C$ & $. q . p 0 .(r, 2)$ & $A^{p} b A b^{q} A C^{r} b C^{s}$ & $. q .(s, r) 1 . p 0$ \\
\hline$A^{p} b A^{q} b A C b C^{r}$ & $. r 11 . p 0 . q$ & $A^{p} b^{q} A^{r} b A C b^{s} C$ & r.q.p. $(2, s) 0$ \\
\hline$A^{p} b A^{q} b^{r} A C b C$ & $.21 . r . q . p 0$ & $A^{p} b^{q} A^{r} b A C b C^{s}$ & r.q.p.s 110 \\
\hline$A^{p} b A b A^{q} C b^{r} C$ & $p 0: q 0:(r, 2) 0$ & $A^{p} b^{q} A^{r} b^{s} A C b C$ & s.r.q.p.210 \\
\hline$A^{p} b A b A^{q} C b C^{r}$ & $r 110: q 0: p 0$ & $A^{p} b^{q} A b A C b^{r} C^{s}$ & $(s 1, r): p: q 0$ \\
\hline$A^{p} b A b A C b^{q} C^{r}$ & $. p .(r 1, q) 0$ & $A^{p} b^{q} A b A C^{r} b C^{s}$ & $(s, r) 1: p: q 0$ \\
\hline$A^{p} b A b A C^{q} b C^{r}$ & $. p .(r, q) 10$ & $A^{p} b^{q} A b^{r} A^{s} C b C$ & s.r.q.p.210 \\
\hline$A^{p} b A b^{q} A^{r} C b C$ & $q .21 . r 0 . p$ & $A^{p} b^{q} A b^{r} A C b^{s} C$ & $p 0 .(s, 2) . q . r 0$ \\
\hline$A^{p} b A b^{q} A C b^{r} C$ & $. q .(2, r) \cdot p 0$ & $A^{p} b^{q} A b^{r} A C b C^{s}$ & $p 0 . s 11 . q . r 0$ \\
\hline$A^{p} b A b^{q} A C b C^{r}$ & .q.r $11 . p 0$ & $A b A^{p} b^{q} A C b^{r} C^{s}$ & $(r, s 1) \cdot q \cdot p$ \\
\hline$A^{p} b^{q} A^{r} b A C b C$ & r.q.p.210 & $A b A^{p} b^{q} A C^{r} b C^{s}$ & $.(s, r) 1 . q . p$ \\
\hline$A^{p} b^{q} A b A C b^{r} C$ & $p:(r, 2): q 0$ & $A b^{p} A^{q} b^{r} A C b^{s} C$ & $(s, 2) \cdot p \cdot r 0 . q 0$ \\
\hline$A^{p} b^{q} A b A C b C^{r}$ & $r 11: p: q 0$ & $A b^{p} A^{q} b^{r} A C b C^{s}$ & $s 1$ 1.r.p $0 . q 0$ \\
\hline$A^{p} b^{q} A b^{r} A C b C$ & $p 0.21 . q . r 0$ & $A b^{p} A b^{q} A C b^{r} C^{s}$ & $q: p:(s 1, r) 0$ \\
\hline$A b A^{p} b A C b^{q} C^{r}$ & $p:(r 1, q)$ & $A b^{p} A b^{q} A C^{r} b C^{s}$ & $p: q:(r, s) 10$ \\
\hline
\end{tabular}

The generating minimum braid $.2: 2$ gives the following $B F R \mathrm{~s}$ :

$\begin{array}{llll}A^{p} b C b A b C b & . p 1: 2 & A^{p} b^{q} C b A b C^{r} b & . r 1 . q 0 . p 1 \\ A b^{p} C b A b C b & .2 . p 0.2 & A^{p} b^{q} C b^{r} A b C b & 2 . q 0 . r . p 10 \\ A^{p} b^{q} C b A b C b & . p 1 . q 0.2 & A^{p} b C b A^{q} b C^{r} b & . r 1:(p, q)\end{array}$




$\begin{array}{llll}A^{p} b C b A^{q} b C b & .(p, q): 2 & A b^{p} C b A b^{q} C b^{r} & 2 . p 0 . r .20 . q \\ A^{p} b C b A b C^{q} b & . p 1: q 1 & A^{p} b^{q} C b A^{r} b^{s} C b & .(p, r) . s 0.2 . q 0 \\ A b^{p} C b A b^{q} C b & . p .20 . q .20 & A^{p} b^{q} C b A^{r} b C^{s} b & . s 1 . q 0 .(p, r) \\ A b^{p} C b A b C b^{q} & 2 . p 0 . q .20 & A^{p} b^{q} C b A^{r} b C b^{s} & (r, p) . q 0 . s .20 \\ A^{p} b^{q} C b A^{r} b C b & .2 . q 0 .(p, r) & A^{p} b^{q} C b A b^{r} C^{s} b & . p 1 . r 0 . s 1 . q 0 \\ A^{p} b^{q} C b A b^{r} C b & . p 1 . r 0.2 . q 0 & A^{p} b^{q} C b A b C^{r} b^{s} & p 1 . s 0 . q . r 10 \\ A^{p} b^{q} C b A b C b^{r} & p 1 . q 0 . r .20 & & \end{array}$

In the same way, from all generating minimum braid words with $s=3$, it is possible to derive alternating and non-alternating $B F R \mathrm{~s}$ and their corresponding families of $K L \mathrm{~s}$.

As the example, the following table contains non-alternating $B F R \mathrm{~s}$ with at most two parameters, derived from the minimum reduced braid $A b A C b C$ :

$\begin{array}{llll}A^{p} B a c B c & (p-1) 32 & A^{p} b a^{q} C b C & p, 22,-q \\ A^{p} B a c B c^{q} & (p-1) 31 q & A^{p} B A^{q} c B c & p, 211,-(q-1) 1 \\ A^{p} b A C B^{q} C & p 1,(q-1) 1,2 & A b^{p} A C B^{q} C & p, 2,2,-q \\ A^{p} B a c B^{q} c & p-1, q, 2++ & A b^{p} A c B^{q} c & p, 2,-q,-2 \\ A^{p} b A C B^{q} C & p 1,(q-1) 1,2 & A b^{p} A c b^{q} c & p, q, 2,-2 \\ A^{p} b A C b c^{q} & p 13(q-1) & A B^{p} A C B^{q} C & p, q,-2,-2 \\ A^{p} B a c B C^{q} & (p-1) 4(q-1) & A B^{p} A C b^{q} C & p, 2, q,-2\end{array}$

From the generating minimum braid word $W=(A b)^{n}(n \geq 2)$, that defines the family of basic polyhedra $(2 n)^{*}$, by word extension $w_{1}=C b A C b C$ we obtain the second family of basic polyhedra $9^{*}(A b A C b A C b C), 10^{* *}(A b A b C b A C b C), 11^{* *}$ $(A b A b A C b A C b C), 12 \mathrm{~F}(A b A b A b C b A C b C)$, etc.

The third family of basic polyhedra $10^{* * *}(A b A b A C b C b C), 11^{* * *}(A b A b A b C b C b C)$, 12I $(A b A b A b A C b C b C)$, etc., is derived from $W=(A b)^{n}(n \geq 3)$ for $w_{1}=C b C b C$.

In the same way, for $W=(A b)^{n}(n \geq 1), w_{1}=C b A b C b A b C b$ the family of basic polyhedra beginning with $12 \mathrm{C}(A b C b A b C b A b C b)$ is obtained;

for $W=(A b)^{n}(n \geq 2), w_{1}=C b A b C b C b$ the family of basic polyhedra beginning with $12 \mathrm{D}(A b A b C b A b C b C b)$ is obtained;

for $W=(A b)^{n}(n \geq 2), w_{1}=C b A b A C b C$ the family of basic polyhedra beginning with $12 \mathrm{G}(A b A b C b A b A C b C)$ is obtained;

for $W=(A b)^{n}(n \geq 2), w_{1}=C b A b C b C$ the family of basic polyhedra beginning with $12 \mathrm{H}(A b A b A C b A b C b C)$ is obtained, etc.

Among them it is possible to distinguish subfamilies obtained using extensions by replacing or by adding.

Theorem 4. For $s=4$ generating algebraic minimum braids are:

$A b A C b d C d, l=8$, with the corresponding link 2222 ,

$A b A b C b d C d, l=9$, with the corresponding link 221112 ,

$A b A b C b C d C d, l=10$, with the corresponding knot 21111112 .

All other generating minimum braid words with $s=4$ are polyhedral.

For $s=4$ and $l \leq 12$, the polyhedral generating braids and their corresponding $K L \mathrm{~s}$ are given in the following table, with the notation for basic polyhedra with 12 crossings according to A. Caudron [3]:

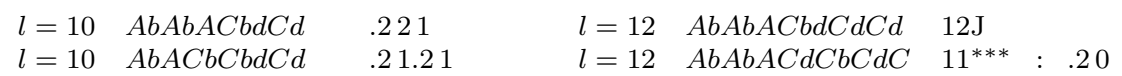




$\begin{array}{llllll}l=10 & A b A C b d C b d C & .21: 210 & l=12 & A b A b C b A b d C b d & 9^{*} 22 \\ l=10 & A b A C d C b C d C & .22: 2 & l=12 & A b A b C b C d C b C d & 8^{*} 211:: 20 \\ & & & l=12 & A b A b C b d C b C d C & 8^{*} 110: .20 \\ l=11 & A b A b A C b C d C d & .21111 & l=12 & A b A b C b d C b d C d & 9^{*} 211 \\ l=11 & A b A b C b C b d C d & .211 .210 & l=12 & A b A b C d C b C d C d & 8^{*} 21110 \\ l=11 & A b A b C b d C b d C & .211: 21 & l=12 & A b A C b A d C b d C d & 12 L \\ l=11 & A b A b C d C b C d C & .2111: 2 & l=12 & A b A C b C b C b d C d & 8^{*} 210.210 \\ l=11 & A b A C b A C b d C d & 9^{*} 210 & l=12 & A b A C b C b d C b C d & 9^{*} .21: .2 \\ l=11 & A b A C b C d C b C d & 8^{*} 210:: 20 & l=12 & A b A C b C b d C b d C & 8^{*} 210: .210 \\ l=11 & A b A C b C d C d C d & .2211 & l=12 & A b A C b C d C b C d C & 9^{*} 21: 2 \\ l=11 & A b A C b d C b C d C & 8^{*} 21: .20 & l=12 & A b A C b C d C b d C d & 10^{* *}: 210 \\ l=11 & A b A C d C b C d C d & 8^{*} 220 & l=12 & A b A C b d C b C d C d & 10^{* *} .21 \\ & & & l=12 & A b A C b d C b d C d C & 10^{* *}: 21 \\ l=12 & A b A b A b A C b d C d & 8^{*} 2210 & l=12 & A b C b A b C d C b C d & 10^{* *}: 20:: 20 \\ l=12 & A b A b A C b A b d C d & 9^{*} .22 & l=12 & A b C b A C b d C b C d & 10^{* *} 20:: .20\end{array}$

For $W=(A b)^{n}(n \geq 2), w_{1}=A C b d C d C d$ the family of basic polyhedra beginning with 12J $(A b A b A C b d C d C d)$ is obtained, and for $W=(A b)^{n}(n \geq 1), w_{1}=$ $A C b A d C b d C d$ the family of basic polyhedra beginning with $12 \mathrm{~L}(A b A C b A d C b d C d)$ is obtained.

\section{Applications of Minimum Braids and $B F R \mathbf{s}$}

\subsection{Graph Trees}

A rational $K L$ in Conway notation is any sequence of natural numbers not beginning or ending with 1 , where each sequence is identified with its inverse. From this definition is computed the number of rational $K L \mathrm{~s}$ with $n$ crossings. It is given by the formula

$$
2^{n-4}+2^{[n / 2]-2}
$$

that holds for every $n \geq 4$. This very simple formula is derived first by C. Ernst and D.W. Sumners in another form [8], and later independently by S. Jablan $[9,10]$. For $n \geq 4$ we can compute the first 20 numbers of this sequence. After prepending to it the first three numbers 1 for $n=1,2,3$, the result is the sequence: 1 , $1,1,2,3,6,10,20,36,72,136,272,528,1056,1080,4160,8256,16512,32986$, $65792,131328,262656,524800, \ldots$ This sequence is included in On-Line Encyclopedia of Integer Sequences (http://www.research.att.com/ njas/sequences/) as the sequence $A 005418$. The number of rational knots with $n$ crossings $(n \geq 3)$ is given by the formula

$$
\frac{2^{n-3}+2^{\left[\frac{n}{2}\right]-2^{(n-1)} \quad(\bmod 2)}+(-1)^{(n-1)\left[\frac{n}{2}\right]} \quad(\bmod 2)}{3}
$$

so we can simply derive the formula for the number of rational links with $n$ crossings as well.

A graph-theoretical approach to knot theory is proposed by A. Caudron [3]. T. Gittings established a mapping between minimum braids with $s$ strands and 
trees with $s+1$ vertices and conjectured that the number of graph trees of $n$ vertices with alternating minimum braids is equal to the number of rational $K L \mathrm{~s}$ with $n$ crossings [5, Conjecture 1].

\subsection{Amphicheiral KLs}

$K L$ is achiral (or amphicheiral) if its "left" and "right" forms are equivalent, meaning that one can be transformed to the other by an ambient isotopy. If an oriented knot or link $L$ can be represented by an antisymmetrical vertex-bicolored graph on a sphere, whose vertices with the sign +1 are white, and vertices with the sign -1 are black, it is achiral. In this case, for an oriented knot or link $L$ there exists an antisymmetry (sign-changing symmetry) switching orientations of vertices, i.e., mutually exchanging vertices with the signs +1 and $-1[9,10]$. In the language of braid words, this means that its corresponding braid word is antisymmetric (or palindromic): there exist a mirror antisymmetry transforming one letter to another and vice versa and changing their case (i.e., transforming capital to lower case letters and vice versa). For example, the reduced braid words $A b \mid A b$ or $A B a c \mid B D c d$ are palindromic, where the anti-mirror is denoted by $\mid$. Hence, we believe that the origin of all oriented achiral $K L \mathrm{~s}$ are palindromic reduced braids.

Conjecture An oriented $K L$ is achiral iff it can be obtained from a palindromic reduced braid by a symmetric assigning of degrees.

For $s=2$ all alternating $B F R$ s are of the form $(A b)^{n}(n \geq 2)$, defining a series of the basic polyhedra $(2 n)^{*}$, beginning with $22, .1=6^{*}, 8^{*}, 10^{*}, 12^{*}$, etc. All of them are achiral $K L \mathrm{~s}$, representing a source of other achiral $K L \mathrm{~s}$. From 4:1-01 $A b A b(22$ or $4_{1}$ ) by a symmetric assigning of degrees we can derive achiral alternating knots with $n \leq 10$ crossings: 6:1-02 $A^{2} b A b^{2}$ (2112 or $6_{3}$ ), 8:1-05 $A^{3} b A b^{3}$ (3113 or 89), 10:1-017 $A^{3} b^{2} A^{2} b^{3}\left((3,2)(3,2)\right.$ or $\left.10_{79}\right)$, and one achiral alternating link with $n \leq 9$ crossings: 8:3-05a $A^{2} b^{2} A^{2} b^{2}\left((2,2)(2,2)\right.$ or $\left.8_{4}^{3}\right)$, etc. In general, from $A b A b$ the following families of achiral alternating $K L$ s are derived:

$A^{p} b A b^{p} \quad p 11 p \quad A^{p} b^{q} A^{q} b^{p} \quad(p, q)(p, q)$

Borromean rings $6: 3-02 A b A b A b\left(.1=6^{*}\right.$ or $\left.6_{2}^{3}\right)$ are the origin of achiral alternating knots 8:1-07 $A^{2} b A b A b^{2}$ (.2.2 or $\left.8_{17}\right), 10: 1-020 A^{2} b A^{2} b^{2} A b^{2}$ (.2.2.2 0.20 or 1099), 10:1-022 $A^{2} b^{2} A b A^{2} b^{2}$ (2.2.2.2 or 10109), and of the link 8:3-04a $A b^{2} A b A^{2} b(.2: 20$ or $8_{6}^{3}$ ), etc. In general, from $A b A b A b$ the following families of achiral alternating $K L \mathrm{~s}$ are derived:

$\begin{array}{llll}A^{p} b A b A b^{p} & . p . p & A^{p} b A^{q} b^{q} A b^{p} & \text {.p.p.q } 0 . q 0 \\ A b^{p} A b A^{p} b & . p: p 0 & A^{p} b^{q} A^{r} b^{r} A^{q} b^{p} & \text { p.q.r.r.q.p } \\ A^{p} b^{q} A b A^{q} b^{p} & \text { p.q.q.p } & & \end{array}$

Achiral basic polyhedron $A b A b A b A b\left(8^{*}\right)$ is the origin of the following families of alternating achiral $K L \mathrm{~s}$ :

$A^{p} b A b A b A b^{p} \quad 8^{*} p \cdot p \quad A^{p} b A b^{q} A^{q} b A b^{p} \quad 8^{*} p . q: . q . p$ 


$\begin{array}{llll}A b A^{p} b A b^{p} A b & 8^{*} p: . p & A^{p} b^{q} A^{r} b A b^{r} A^{q} b^{p} & 8^{*} \text { p.q.r.r.q.p } \\ A^{p} b^{q} A b A b A^{q} b^{p} & 8^{*} \text { p.q.q.p } & A^{p} b A^{q} b^{r} A^{r} b^{q} A b^{p} & 8^{*} \text { p.q.q.p }: \text { r.r } \\ A^{p} b A^{q} b A b^{q} A b^{p} & 8^{*} . p: q . q: p & & \end{array}$

In the same way it is possible to derive achiral alternating $K L$ s from all achiral basic polyhedra $(A b)^{n}$ for $n \geq 5$.

From the antisymmetry condition it follows that every palindromic braid has an even number of strands. For $s=4$ and $l \leq 12$ palindromic algebraic generating braids are:

$A b A C b d C d, l=8$ with the corresponding achiral link 2222 ,

$A b A b C b C d C d, l=10$, with the corresponding achiral knot 21111112 .

The palindromic polyhedral generating braids are:

$A b A C b C b d C d, l=10$, with the corresponding achiral knot .21.21,

$A b A b A C b d C d C d, l=12$, with the corresponding achiral link 12J,

$A b A C b A d C b d C d, l=12$, with the corresponding achiral knot $12 \mathrm{~L}$,

$A b A C b C b C b d C d, l=12$, with the corresponding achiral link $8^{*} 210.210$,

$A b C b A b C d C b C d, l=12$, with the corresponding achiral knot $10^{* *}: 20:: .20$,

$A b C b A C b d C b C d, l=12$, with the corresponding achiral knot $10^{* *} 20:: .20$.

From the generating braid $A b A C b d C d$ following families of alternating achiral $K L \mathrm{~s}$ are derived:

$\begin{array}{llll}A^{p} b A C b d C d^{p} & p 1221 p & A^{p} b A^{q} C^{r} b^{r} d^{q} C d^{p} & (((p, q), r)+)(((p, q), r)+) \\ A b A C^{p} b^{p} d C d & (p, 2+)(p, 2+) & A^{p} b^{q} A C^{r} b^{r} d C^{q} d^{p} & (q, p 1, r)(q, p 1, r) \\ A b^{p} A C^{q} b^{q} d C^{p} d & (p, q, 2)(p, q, 2) & A^{p} b^{q} A^{r} C^{s} b^{s} d^{r} C^{q} d^{p} & (q,(p, r), s)(q,(p, r), s)\end{array}$

From the same palindromic non-alternating generating braid the following families of achiral $K L \mathrm{~s}$ are obtained:

$\begin{array}{llll}A^{p} B a c B D c d^{p} & p p & A^{p} B a c B D c d^{p} & 2 p p 2 \\ A b A c^{p} B^{p} d C d & (p, 2)(q, 2) & A^{p} b A c^{q} B^{q} d C d^{p} & (p 1, q)(p 1, q)\end{array}$

In the same way is possible to continue the derivation of achiral $K L$ s from other palindromic reduced braids.

The family of achiral odd crossing number knots discovered by J. Hoste, M. Thistlethwaite and J. Weeks in 1998 [11] can be extended to the two-parameter BFR defined by the palindromic braid $A B a B^{q} C^{p} B A d c b^{p} c^{q} D c d$ corresponding to the family of non-alternating achiral odd-crossing knots with $n=7+4 p+4 q$ crossings

$$
10^{* *}(-2 p) 0 .-1 .-20 .(2 q):(-2 p) 0 .-1 .-20 .(2 q)
$$

\subsection{Unlinking Numbers and Unlinking Gap}

T. Gittings [5] noticed that it might be possible to calculate unlinking numbers from minimum braids. Unfortunately, this is true only for $K L$ s with $n \leq 10$ crossings, including the link $414\left(9_{4}^{2}\right)$ and the Nakanishi-Bleiler example $514\left(10_{8}\right)$ with an unlinking gap [12]. 
Definition 7. The minimum braid unlinking gap is the positive difference between the unlinking number obtained from a minimum braid $u_{B}(L)$ and unlinking number $u(L)$ of a link $L$, i.e.,

$$
\delta_{B}=u_{B}(L)-u(L)>0 .
$$

The unlinking gap [12] for minimum braids appears for $n=11$. The following alternating links given in Conway notation, followed by their minimum braids have the minimum braid unlinking gap:

$\begin{array}{llll}.5 .2 & A^{5} b A b A b^{2} & 8^{*} 3.2 & A^{3} b A b A b A b^{2} \\ .3 .4 & A^{4} b A b A b^{3} & 8^{*} 3: 2 & A^{3} b A^{2} b A b A b \\ 8^{*} 4 & A^{4} b A b A b A b & 8^{*} 2.2: .2 & A^{2} b A b A^{2} b A b^{2} \\ .2 .3 .30 & A^{3} b A^{3} b A b^{2} & 10^{*} 2 & A^{2} b A b A b A b A b\end{array}$

For the links $.5 .2, .3 .4$ the value of minimum braid unlinking gap is $\delta_{B}=2$, and for other links from this list $\delta_{B}=1$. Hence, minimum braid unlinking number is different from the unlinking number and represents a new $K L$ invariant.

\subsection{Periodic Tables of KLs}

Periodic tables of $K L$ s can be established in three ways: starting with families of $K L$ s given in Conway notation [9,10,13], with minimum braids [5], or with $B F R$ s. Since we have established correspondence between $B F R$ s and $K L \mathrm{~s}$ in Conway notation, it follows that the same patterns (with regard to all $K L$ polynomial invariants and $K L$ properties) will appear in all cases. For example, for every family of $K L \mathrm{~s}$ is possible to obtain a general formula for Alexander polynomials, with coefficients expressed by numbers denoting tangles in Conway symbols, or from their corresponding parameters from minimum braids or from $B F R \mathrm{~s}$. The same holds not only for $K L$ polynomials, but for all other properties of $K L \mathrm{~s}$ : writhe, amphicheirality, number of projections, unlinking number, signature, periods, etc. $[9,10,13]$.

\section{Acknowledgements}

We would like to express our gratitude to Thomas Gittings for his critical reading of the manuscript, corrections, advice and suggestions.

\section{References}

[1] C. C. Adams, The Knot Book, Freeman, New York (1994).

[2] J. Conway, An enumeration of knots and links and some of their related properties, in Computational Problems in Abstract Algebra, Proc. Conf. Oxford 1967 (Ed. J. Leech), 329-358, Pergamon Press, New York (1970).

[3] A. Caudron, Classification des nœuds et des enlancements, Public. Math. d'Orsay 82. Univ. Paris Sud, Dept. Math., Orsay (1982). 
[4] D. Rolfsen, Knots and Links, American Mathematical Society, AMS Chelsea Publishing (2003).

[5] T. Gittings, Minimum braids: a complete invariant of knots and links, (preprint).

[6] S. Jablan and R. Sazdanović , LinKnot, http://www.mi.sanu.ac.yu/vismath/linknot/ (2003).

[7] M. Ochiai and N. Imafuji , Knot2000, http://amadeus.ics.nara-wu.ac.jp/ ochiai/freesoft.html

[8] C. Ernst and D. W. Sumners The growth of the number of prime knots, Math. Proc. Cambridge Math. Soc. 102 (1987), 303-315.

[9] S. V. Jablan, Ordering knots, Visual Mathematics 1, 1 (1999), http://members.tripod.com/vismath/sl/index.html

[10] S. V. Jablan, Geometry of links, Novi Sad J. Math. 29, 3 (1999), 121-139.

[11] J. Hoste, M. Thistlethwaite and J. Weeks, The first 1,701,936 knots, Math. Intalligencer 20 (1998), 33-48.

[12] S. Jablan and R. Sazdanović, Unlnking number and unlikng gap, (to appear).

[13] S. V. Jablan, New knot tables, Filomat (Niš), , (2002), 141-152 (http://members.tripod.com/modularity/knotab/index.html). 